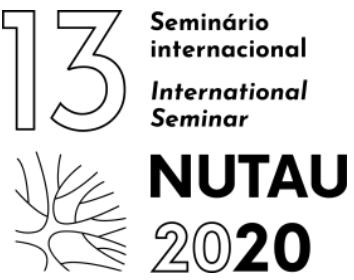

\title{
Reaproveitamento de resíduos de poda e sua colaboração para atingir os Objetivos de Desenvolvimento Sustentável
}

Caroline Almeida Souza ${ }^{1}$, Camila Camolesi Guimarães ${ }^{2}$, Giuliana Del Nero Velasco ${ }^{3}$

${ }^{1}$ Instituto de Pesquisas Tecnológicas, MSc., caroline@ipt.br

2 Instituto de Pesquisas Tecnológicas, MSc., camilacg@ipt.br

3 Instituto de Pesquisas Tecnológicas, Dr., velasco@ipt.br

\begin{abstract}
RESUMO: O objetivo do presente artigo é apresentar uma análise dos potenciais ODS associados às principais opções de reaproveitamento de resíduos de poda de arborização urbana, indicadas na literatura científica, de forma a indicar as formas de reaproveitamento que promovam maior contribuição para o atingimento de metas dos ODS pelos municípios brasileiros. Para o levantamento e análise bibliográfica de opções de reaproveitamento, foram realizadas buscas nas principais bases de dados nacionais, sendo obtidos 11 resultados relevantes para o presente estudo. Foram identificados 12 principais produtos que podem ser gerados pelo reaproveitamento de resíduos de poda de arborização urbana, relacionados aos setores de construção civil, agricultura/jardinagem, energia, decoração/materiais pedagógicos e esportivos e indústria química. Analisando-se os produtos estudados e considerados nesse trabalho, verifica-se que o composto orgânico apresenta o maior potencial de colaboração para o atendimento aos ODS, estando associado a nove metas distribuídas em cinco ODS. Concluiu-se que os ODS são bons indicativos para a definição de possíveis usos para os resíduos de poda de arborização urbana nos municípios. Deve-se, porém, ser realizada uma análise de viabilidade técnica, social, econômica e ambiental para os usos de interesse, de forma a selecionar os que melhor se aplicam à realidade do município.
\end{abstract}

PALAVRAS-CHAVE: Resíduos, arborização, sustentabilidade, reaproveitamento, Objetivos do Desenvolvimento Sustentável.

\begin{abstract}
The purpose of this article is to present an analysis of the potential SDGs associated with the main options for reusing urban forest pruning waste, indicated in the scientific literature, in order to indicate the forms of reuse that promote a greater contribution to the achievement of SDG targets by Brazilian municipalities. For the survey and bibliographic analysis of reuse options, searches were carried out in the main national databases, obtaining 11 results relevant to the present study. 12 main products were identified that can be generated by the reuse of pruning residues from urban forest, related to the sectors of civil construction, agriculture/gardening, energy, decoration/educational and sports materials and the chemical industry. Analysing the products studied and considered in this work, it appears that the organic compost has the greatest potential for collaboration to meet the SDGs, being associated with nine goals distributed in five SDGs. It was concluded that the SDGs are good indicators for the definition of possible uses for urban forest pruning residues in the municipalities. However, an analysis of the technical, social, economic and environmental feasibility must be carried out for the uses of interest, in order to select those that best apply to the reality of the municipality.
\end{abstract}

KEYWORDS: Waste, Urban Forest, Sustainability, Reuse, Sustainable Development Goals

\section{INTRODUÇÃO}

Segundo Santos (2000), na arborização urbana a poda é utilizada para adequar a planta ao interesse do homem que habita a cidade, sendo, desta forma, executada para corrigir os conflitos existentes entre as árvores e os equipamentos e/ou edificações da cidade. Dessa maneira, a poda em árvores urbanas é uma prática muito comum e a quantidade de resíduos gerada resultante de tal prática, é preocupante, sendo de extrema importância a avaliação das melhores técnicas de reaproveitamento de resíduos de acordo com sua composição. Segundo Rocha et al. (2015), a Prefeitura Municipal de São Paulo estima recolher de 3,5 a 4 mil toneladas de resíduos da poda de árvores por mês. 0 volume anual pode chegar a 50 mil toneladas (galhos e troncos).

Os resíduos de poda provenientes da arborização urbana apresentam características diversas de acordo com sua composição (ex.: troncos, galhos e raízes) e espécie vegetal. Dependendo das características dos resíduos gerados, opções de reaproveitamento podem ser elencadas, podendo gerar materiais com realização organized by apoio institucional supported by
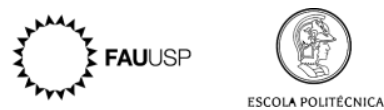
diversos graus de processamento, como lenha, carvão, pequenos objetos de madeira e painéis de madeira. O processamento envolvido nas diversas opções de reaproveitamento e os produtos gerados promovem diferentes benefícios econômicos, sociais e ambientais. Assim, a tomada de decisão sobre como reaproveitar os resíduos de poda de uma localidade requer uma avaliação desses benefícios para que a melhor opção seja escolhida.

Nesse sentido, os Objetivos do Desenvolvimento Sustentável (ODS), estabelecidos pela Organização das Nações Unidas (ONU), podem embasar uma abordagem de avaliação das opções de reaproveitamento de resíduos de poda. Os ODS, também conhecidos como Objetivos Globais, foram adotados pelos países membros da ONU em 2015 e tem por premissa a orientação das políticas nacionais e atividades de cooperação internacional até 2030, com base no combate à pobreza, proteção do planeta e garantia da paz e prosperidade (Programa das Nações Unidas para o Desenvolvimento [PNUD], 2020). Foram estabelecidos 17 ODS com 169 metas a serem alcançadas por meio de ação conjunta que agrega diferentes níveis de governo, organizações, empresas e sociedade, nos âmbitos internacional, nacional e local (Confederação Nacional dos Municípios [CNM], 2020).

Os 17 ODS estão organizados em cinco áreas principais: pessoas (erradicar a pobreza e a fome de todas as maneiras e garantir a dignidade e a igualdade); prosperidade (garantir vidas prósperas e plenas, em harmonia com a natureza); paz (promover sociedades pacíficas, justas e inclusivas); parcerias (implementar a agenda por meio de parceria global sólida); e planeta (proteger os recursos naturais e o clima do planeta para as futuras gerações). Os temas abordados são cruciais para a promoção do desenvolvimento sustentável dos municípios, considerando os aspectos sociais, ambientais e econômicos, sendo os ODS uma ferramenta importante para o planejamento das ações municipais. Como colocado pela CNM (2020), "os municípios tem um papel central no sucesso dessa agenda, pois, para que os ODS sejam disseminados e alcançados, é preciso que os gestores municipais incluam tais objetivos em suas políticas e projetos, promovam a integração e a sustentabilidade das iniciativas e atuem a partir de acordos e articulação com outros agentes territoriais".

Nesse contexto, observa-se que a maioria dos estudos sobre o reaproveitamento de resíduos de poda de arborização urbana não associam os ODS na avaliação das opções analisadas. Estudos mais recentes, como os realizados por Bispo (2017) e Ribaski \& Belini (2019), já fazem essa associação, porém não contemplam todos os ODS potencialmente associados às opções de reaproveitamento analisadas. Com o intuito de colaborar para o desenvolvimento de uma metodologia de avaliação de opções de reaproveitamento de resíduos de poda com base nos ODS, o objetivo do presente artigo é apresentar uma análise dos potenciais ODS associados às principais opções de reaproveitamento indicadas na literatura científica, de forma a indicar as formas de reaproveitamento de resíduos de arborização urbana que promovam maior contribuição para o atingimento de metas dos ODS pelos municípios brasileiros.

\section{METODOLOGIA}

O presente estudo foi realizado em três etapas: levantamento e análise bibliográfica sobre as principais opções de reaproveitamento de resíduos de poda de arborização urbana; levantamento dos ODS e metas relacionadas à adoção de cada opção de reaproveitamento; e identificação dos benefícios associados às opções de reaproveitamento, relacionados ao cumprimento das metas identificadas. Para o levantamento e análise bibliográfica de opções de reaproveitamento, foram realizadas buscas nas principais bases de dados nacionais, por meio do Portal de Periódicos da Capes, utilizando as seguintes palavras-chave: árvore urbana (urban tree), poda (pruning), arborização urbana (urban afforestation), uso de resíduos de árvores (tree waste/residues use/utilization), logística (logistics) e reciclagem (recycling). Foram obtidos 13 resultados, sendo 11 relevantes para o presente trabalho, dentre artigos de periódicos, dissertações e teses, sendo considerados como adequados ao objetivo do estudo e utilizados para a análise. A partir dessa análise, foram elencados os principais usos possíveis para reaproveitamento de resíduos de arborização urbana municipais.

Após o levantamento e análise bibliográfica, foram analisados os 17 ODS e as respectivas 169 metas, de forma a correlacioná-las com os principais usos levantados. Para cada uso, foram selecionadas as metas para as quais estes apresentam contribuição direta, caso implantados nos municípios. Por fim, foram listados, para as metas dos ODS identificadas, os benefícios socioambientais decorrentes dos tipos de reutilização possíveis para os resíduos de arborização urbana, considerando-se como usos mais desejáveis aqueles que contribuem para o cumprimento de um maior número de metas, de acordo com a realidade dos municípios.

\section{RESULTADOS E DISCUSSÃO}

Com base em Cortez (2011), Luz (2012), Silva, et al. (2013), Martins (2013), Oliveira e Bariccatti (2016), Bispo (2017), Rocha (2017), Palharini, et al. (2018), Ribaski e Belini (2019), Silva et al. (2019) e Cruz- 
Estrada et al. (2020), os 12 principais produtos que podem ser gerados pelo reaproveitamento de resíduos de poda de arborização urbana são relacionados a cinco setores econômicos:

1. Construção civil

a. Produtos sólidos de madeira: fabricação de móveis, portas, guarnições, vigas, tábuas, assoalhos e outros;

b. Painéis de madeira: fabricação de painéis de madeira reconstituída (com processamento químico da madeira) e painéis de madeira processada mecanicamente;

c. Agregado para confecção de blocos de concreto: utilização do pó de serra como agregado miúdo na confecção de blocos de concreto;

d. Compósitos termoplástico-celulósicos para fabricação de materiais de construção.

2. Agricultura/jardinagem

a. Composto orgânico: obtido pelo processo de compostagem;

b. Cama de aves: utilização de serragem;

c. Biomassa para proteção do solo: utilização de serragem e madeira sólida picada in natura.

3. Energia

a. Biocombustíveis sólidos para produção de energia elétrica e térmica: produção de lenha, carvão vegetal e produtos densificados de madeira (lascas, briquetes e pellets);

b. Bioetanol: obtido por meio da hidrólise ácida da biomassa lignocelulósica e fermentação de açúcares;

c. Gaseificação da madeira para geração de energia elétrica e térmica.

4. Decoração, design, materiais pedagógicos e esportivos

a. Pequenos objetos de madeiras (POM): fabricação de artigos domésticos, peças decorativas, equipamentos esportivos, objetos artesanais e brinquedos educativos.

5. Indústria química

a. Resinas plásticas, colas e essências: obtidas com a extração de óleos e resinas dos resíduos de serragem.

Foi identificada possível contribuição das opções de uso dos resíduos de poda de arborização urbana para os ODS e metas apresentados no Quadro 1, resumidos de forma a evidenciar as contribuições dos usos estudados.

\section{ODS 2: Fome Zero e Agricultura Sustentável}

Meta 2.1: “(...) acabar com a fome e garantir o acesso de todas as pessoas (...) a alimentos seguros, nutritivos e suficientes (...)".

Meta 2.3: “(...) dobrar a produtividade agrícola e a renda dos pequenos produtores de alimentos (...) inclusive por meio de acesso (...) a outros recursos produtivos e insumos (...)".

Meta 2.4: “(...) garantir sistemas sustentáveis de produção de alimentos e implementar práticas agrícolas resilientes (...) e que melhorem progressivamente a qualidade da terra e do solo".

\section{ODS 6: Água Potável e Saneamento}

Meta 6.3: (...) melhorar a qualidade da água, reduzindo a poluição, (...) minimizando a liberação de produtos químicos e materiais perigosos (...)".

\section{ODS 7: Energia Limpa e Acessível}

Meta 7.2: “(...) aumentar substancialmente a participação de energias renováveis na matriz energética global (...)".

ODS 8: Trabalho Decente e Crescimento Econômico

Meta 8.5: "(...) alcançar o emprego pleno e produtivo e trabalho decente para todas as mulheres e homens (...)".

Meta 8.6: “(...) reduzir a proporção de jovens sem emprego, educação ou formação (...)".

ODS 10: Redução das Desigualdades

Meta 10.2: “(...) empoderar e promover a inclusão social, econômica e política de todos (...)".

ODS 11: Cidades e Comunidades Sustentáveis

Meta 11.6: “(...) reduzir o impacto ambiental negativo per capita das cidades, prestando especial atenção à (...) gestão de resíduos municipais (...)".

ODS 12: Consumo e Produção Responsáveis

Meta 12.2: “(...) alcançar a gestão sustentável e uso eficiente dos recursos naturais".

Meta 12.4: “(...) alcançar o manejo ambientalmente saudável (...) de todos os resíduos (...), e reduzir significativamente a liberação destes para o ar, água e solo (...)".

Meta 12.5: “(...) reduzir substancialmente a geração de resíduos por meio da prevenção, redução, reciclagem e reuso".

\section{ODS 15: Vida Terrestre}

Meta 15.3: “(...) restaurar a terra e o solo degradado, (...) e lutar para alcançar um mundo neutro em termos de degradação de solo". 
Quadro 1 -ODS e respectivas metas identificadas, relacionadas ao reaproveitamento de resíduos de arborização urbana

Pensando no reaproveitamento de resíduos de arborização urbana, de uma forma geral, independente do produto gerado, foi identificada contribuição para o cumprimento dos ODS 11 e 12, especificamente das metas 11.6, 12.2, 12.4 e 12.5, por estarem relacionadas à reutilização de resíduos e uso eficientes de recursos naturais. Além da colaboração a essas metas, apresentam-se, a seguir, a correlação entre as opções de usos possíveis para os resíduos de arborização urbana identificadas e os ODS e metas levantados, destacando-se os benefícios socioambientais decorrentes desses usos:

1. Construção civil

a. Produtos sólidos de madeira para a construção civil - possível contribuição para ODS 8 (metas 8.5 e 8.6), por estar associado à fabricação de novos produtos de maior valor agregado com base em matéria-prima advinda de resíduos, sendo alternativa de geração de emprego e renda com o estabelecimento de pequenos negócios;

b. Painéis de madeira - possível contribuição para ODS 8 (metas 8.5 e 8.6), por estar associado à fabricação de novos produtos de maior valor agregado com base em matéria-prima advinda de resíduos, sendo alternativa de geração de emprego e renda com 0 estabelecimento de pequenos negócios;

c. Agregado para confecção de blocos de concreto - não foi identificada contribuição direta para o cumprimento de outros ODS, além dos ODS 11 e 12 (metas 11.6, 12.2, 12.4 e 12.5) relacionados ao reaproveitamento de resíduos de arborização urbana em geral;

d. Compósitos termoplástico-celulósicos para fabricação de materiais de construção - possível contribuição para o ODS 8 (metas 8.5, 8.6) e ODS 10 (meta 10.2), por estar associado a: fabricação de materiais de construção com menor custo e melhor desempenho técnico que materiais de baixa qualidade utilizados atualmente por populações de baixa renda, sendo alternativa para atender esse público; e geração de emprego e renda com 0 estabelecimento de pequenos negócios.

2. Agricultura/jardinagem

a. Composto orgânico - possível contribuição para os ODS 2 (Metas: 2.1, 2.3 e 2.4), ODS 6 (meta 6.3) e ODS 15 (meta 15.3), por estar associado a: uso do composto como condicionador de solo na agricultura; redução de gastos com adubação de praças públicas; substituição da adubação química;

b. Cama de aves - possível contribuição para ODS 2 (meta 2.4), por estar associado à redução de custos com aquisição de serragem na avicultura;

c. Biomassa para proteção do solo - possível contribuição para ODS 2 (meta 2.4) e ODS 15 (meta 15.3), por estar associado à conservação do solo na agricultura e em áreas verdes.

3. Energia

a. Biocombustíveis sólidos para produção de energia elétrica e térmica - possível contribuição para os ODS 7 (meta 7.2), por oferecer alternativa competitiva para o uso de combustíveis fósseis, com menores custos relacionados ao transporte e logística e a melhoria das propriedades de queima, minimizando a emissão de material particulado;

b. Bioetanol - possível contribuição para ODS 2 (metas 2.1, 2.3, 2.4); ODS 7 (meta 7.2), por oferecer alternativa competitiva para o uso de combustíveis fósseis e por reduzir a competição por área para plantios voltados para a produção de alimentos e para a produção de biocombustíveis;

c. Gaseificação da madeira para geração de energia elétrica e térmica - ODS 7 (meta 7.2), por oferecer alternativa competitiva para o uso de combustíveis fósseis.

4. Decoração/materiais pedagógicos e esportivos

a. POM - possível contribuição para o ODS 8 (metas 8.5, 8.6) e ODS 10 (meta 10.2), por estar associado a: geração de emprego e renda, com possibilidade de empregar mão de obra de comunidades carentes; melhoria da economia local; apoio ao empreendedorismo, com a atração de pequenos investidores e incentivando a cultura tradicional; aproveitamento econômico de resíduos, com baixo custo de obtenção de matériaprima (muitas vezes gratuita); fabricação de produtos diversos acessíveis a várias faixas de renda.

5. Indústria química

a. Resinas plásticas, colas e essências possível contribuição para ODS 8 (metas 8.5 e 8.6), por estar associado à fabricação de novos produtos de maior valor 
agregado com base em matéria-prima advinda de resíduos, sendo alternativa de geração de emprego e renda com o estabelecimento de pequenos negócios.

Percebe-se que os resíduos de madeira de poda podem ser aproveitados em diversos setores econômicos, sendo que seu uso tem sido estudado para aplicação na agricultura, jardinagem, decoração, produção de materiais pedagógicos e esportivos, construção civil, energia e indústria química. Isso demonstra o potencial e a oportunidade de utilização dos restos de poda que, ao invés de serem dispostos em aterro como resíduos sólidos, podem ser considerados como matéria prima para a fabricação de novos produtos, aliando geração de emprego e renda ao uso eficiente de recursos naturais.

Analisando-se os produtos estudados e considerados nesse trabalho, verifica-se que o composto orgânico apresenta o maior potencial de colaboração para o atendimento aos ODS, estando associado a nove metas distribuídas em cinco ODS. Em segundo lugar está o bioetanol, com o potencial de atender a oito metas distribuídas em quatro ODS. Em terceiro lugar estão os POM e os compósitos termoplástico-celulósicos, ambos associados a sete metas distribuídas em quatro ODS. A biomassa para proteção do solo aparece em quarto lugar, associada a seis metas distribuídas em quatro ODS. O quinto lugar é ocupado por três produtos associados a seis metas distribuídas em três ODS: produtos sólidos de madeira para a construção civil; painéis de madeira; e resinas plásticas, colas e essências. A sexta posição também inclui três produtos associados a cinco metas distribuídas em três ODS: cama de aves; biocombustíveis sólidos para produção de energia elétrica e térmica; e gaseificação da madeira para geração de energia elétrica e térmica. Em último lugar está o agregado para confecção de blocos de concreto, associado a quatro metas distribuídas em dois ODS.

Porém, a tomada de decisão por parte do gestor público sobre quais as melhores opções de reaproveitamento de resíduos de poda deve considerar outros fatores relacionados com os desafios ambientais, sociais e econômicas enfrentados pelo município. É importante avaliar quais ODS o município necessita dar maior atenção, relacionando às melhores opções de reutilização dos resíduos de poda para atendê-los (Figura 1).

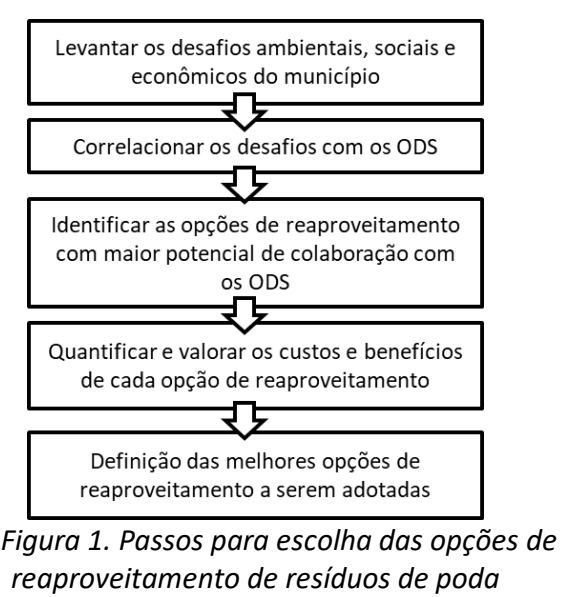

O resultado dessa análise pode ser refinado por meio da aplicação de outros métodos de análise que envolvam a quantificação de benefícios ambientais, como o cálculo da pegada de carbono e da pegada hídrica e a Avaliação de Ciclo de Vida (ACV), que poderão indicar, por exemplo, os benefícios de cada alternativa de reaproveitamento para a redução de emissões de gases de efeito estufa, de consumo de água e energia. Essas análises permitirão a comparação das opções de reutilização consideradas entre si e entre elas e a destinação atual desses resíduos. Da mesma forma, a Análise Custo-Benefício também pode ser conduzida para quantificar os benefícios socioeconômicos e valorar os benefícios ambientais e socioeconômicos. Esse tipo de análise permite comparar os valores dos benefícios identificados nas alternativas de reaproveitamento consideradas e aos resultados da mesma análise para a situação atual de destinação dos resíduos da poda da arborização urbana. Exemplos de benefícios ambientais a compor essa análise inclui a valoração dos benefícios da não disposição dos resíduos em aterros sanitários, relacionados ao aumento da vida útil desses aterros. No caso de benefícios socioeconômicos a incluir na análise pode-se citar o aumento da arrecadação de impostos relacionados à comercialização dos produtos resultantes do reaproveitamento dos resíduos de poda. Já no caso de benefícios sociais a incluir na análise pode-se citar a redução na taxa de desemprego e a inclusão social da população vulnerável do município.

\section{CONCLUSÃO}

Este trabalho apresentou uma análise das opções de reaproveitamento de resíduos de poda levantadas em estudos recentes para orientar a tomada de decisão dos gestores municipais, com base no atendimento aos ODS. Conclui-se que os ODS são bons indicativos para a definição de possíveis usos para os resíduos de poda de arborização urbana nos municípios. Deve-se, porém, ser realizada uma análise de viabilidade técnica, social, econômica e ambiental para os usos de interesse, de forma a 
selecionar os que melhor se aplicam à realidade do município.

\section{AGRADECIMENTOS}

Ao Instituto de Pesquisas Tecnológicas pelo financiamento do projeto "Soluções Técnicas para gestão de resíduos de árvores urbanas de Bertioga/SP" e à toda a equipe envolvida.

\section{REFERÊNCIAS}

1. Bispo, L. F. P. (2017). Aproveitamento de resíduos da arborização urbana para fabricação de Brinquedos [Trabalho de Conclusão de Curso, Universidade de São Paulo].

2. Confederação Nacional dos Municípios (2020, 02 de outubro). Agenda 2030 para o Desenvolvimento Sustentável. https://ods.cnm.org.br/agenda2030\#oQueEAgenda.

3. Cortez, C. L. (2001). Estudo do potencial de utilização da biomassa resultante da poda de árvores urbanas para a geração de energia: estudo de caso: AES Eletropaulo. [Tese de Doutorado, Universidade de São Paulo].

4. Cruz-Estrada, R. H., Guillén-Mallette, J., Cupul-Manzano, C. V., \& Balam-Hernández, J. I. (2020). Potential use of waste from tree pruning and recovered plastic to obtain a building material: Case study of Merida, Mexico. Waste Management \& Research, $0734242 \times 20928404$.

5. Luz, S. (2012). Aproveitamento de madeiras de podas da arborização urbana: áreas do traçado inicial de Maringá/PR. [Dissertação de Mestrado, Universidade Estadual de Maringá].

6. Martins, C. H. (2013). O aproveitamento de madeiras das podas da arborização viária de Maringá / PR. Revista Verde de Agroecologia e Desenvolvimento Sustentável, 8 (2), 257267.

7. Nolasco, A. M.; Meira, A. M.; Gatti, R. C. (2013). Capacitação de gestores públicos em gerenciamento de resíduos da arborização urbana. Revista de Cultura $e$ Extensão USP, 9, 65-73.

8. Oliveira, F. A. de. (2016). Aproveitamento da biomassa residual urbana para síntese de bioetanol. [Dissertação, Universidade Estadual do Oeste do Paraná].

9. Palharini, K. M. Z., Junior, J. B. G., Faria, D. L., Mendes, R. F., de Paula Protásio, T., \& Mendes, L. M. (2018). Potential usage of the urban pruning residue for production of wood based panels. Nativa: Pesquisas Agrárias e Ambientais, 6(3), 321-325.

10. Programa Das Nações Unidas Para O Desenvolvimento (2020, 02 de outubro). Objetivos de Desenvolvimento Sustentável.

https://www.br.undp.org/content/brazil/pt/home/sustaina ble-development-goals.html.

11. Ribaski, N. G., Belini, H. L. (2019). Aproveitamento de resíduos sólidos urbanos madeireiro. Brazilian Journal of Technology, 2 (3), 742-757.

12. Rocha, A. J. F., de Souza, R. L. P., de Lima Reda, A. L., \& da Silva, G. T. Destinação sustentável do resíduo da poda de árvores urbanas. XV Safety, Health and Environment World Congress, 19 (22), 137-141.

13. Rocha, C. T. (2017). Avaliação do potencial de uso dos resíduos de madeira e borra oleosa para produção de briquetes. [Dissertação, Universidade Estadual de Lodrina].
14. Santos, E. dos. (2000). Avaliação quali-quantitativa da arborização e comparação econômica entre a poda e a substituição da rede de distribuição de energia elétrica da região administrativa Centro - Sul de Belo Horizonte - MG. [Tese, Universidade Federal de Viçosa].

15. Silva, A. C. da., Paiva, B. K. V., Sá, G. G., De Carvalho, R. L. T., \& Leite, N. D. (2019). Gerenciamento dos resíduos de poda urbana no município de Fortaleza-Ceará. 10ํㅡórum Internacional de Resíduos Sólidos.

16. Silva, L. S.; Freire, W. R.; Jr.; Basseto, L. I. (2012). Mercado de carbono e instituições: Oportunidades na busca por um novo modelo de desenvolvimento. Interciência, 37, 8-13.

17. Duarte da Silva, M. J., Bezerra, B. S., Gomes Battistelle, R. A., \& De Domenico Valarelli, I. (2013). Prospects for the use of municipal tree pruning wastes in particleboard production. Waste management \& research,31(9), 960965 . 